\title{
Efeitos adversos e resposta citogenética em pacientes com leucemia mieloide crônica tratados com imatinibe
}

\author{
Adverse events and cytogenetc response in patients with chronic myeloid leukemia \\ treated with imatinib
}

\author{
Tatiana F. Alvarenga ${ }^{I}$ \\ Luize O. Carvalho ${ }^{2}$ \\ Stella B. Lucenas ${ }^{3}$ \\ Jane Dobbins ${ }^{4}$ \\ Alexandre Azevedo 5 \\ Teresa S. Fernandez ${ }^{6}$ \\ Maria Helena Ornellas ${ }^{7}$
}

\begin{abstract}
A leucemia mieloide crônica (LMC) é uma doença mieloproliferativa clonal caracterizada citogeneticamente pelo cromossomo Philadelphia. Dentre as opções terapêuticas estão a hidroxiureia, o interferon- $\alpha$, o transplante alogeneico de células-tronco hematopoéticas e o imatinibe. Esta última terapia tem demonstrado eficácia, principalmente na fase crônica da doença. Entretanto, alguns estudos têm demonstrado que alterações cromossômicas adicionais levam resistência à terapia, enquanto outros relatam aparecimento de manifestações clínicas indesejáveis, como cefaleia, náuseas e vômitos. Devido à importância desta terapia alvo-molecular, torna-se necessário analisar a resposta deste tratamento considerando a qualidade de vida dos pacientes. O objetivo deste trabalho foi analisar as manifestações clínicas indesejáveis e a resposta citogenética durante o tratamento com imatinibe em pacientes com LMC após uso prévio de interferon- $\alpha$. O estudo clínico foi feito através de prontuários de 51 pacientes. A análise citogenética foi feita em células de medula óssea através da técnica de bandeamento GTG. As manifestações clínicas mais frequentes foram: cefaleia (37\%), náusea (37\%), vômito (33\%) e edema periférico (33\%). Esses sintomas foram considerados leves a moderados. Os pacientes que alcançaram resposta citogenética completa tiveram uma sobrevida significativamente maior que os pacientes que não apresentaram resposta citogenética ao tratamento $(p=0.007)$. Oito pacientes sem resposta citogenética faleceram. Nossos resultados mostraram a importância do acompanhamento clínico (analisando o grau de tolerância medicamentosa) e citogenético, onde a presença de alterações cromossômicas adicionais mostrou um comportamento biológico distinto que não pode ser avaliado pelas técnicas moleculares. Desta forma, a análise citogenética representa uma importante ferramenta para o diagnóstico e monitoramento destes pacientes. Rev. Bras. Hematol. Hemoter. 2010;32(2):116-122.
\end{abstract}

Palavras-chave: Leucemia mieloide; sinais e sintomas; análise citogenética. \footnotetext{
FCM-Uerj - Rio de Janeiro-RJ.

Correspondência: Maria Helena Ornellas

Faculdade de Ciências Médicas, Disciplina de Patologia Geral

Avenida Professor Manuel de Abreu 444, $4^{\circ}$ andar, Vila Isabel

20550-170 - Rio de Janeiro-RJ - Brasil

E-mail: mariahelenaornellas@ig.com.br

Doi: $10.1590 /$ S1516-84842010005000028
}

${ }_{2}^{l}$ Médica. Residente de Patologia da Divisão de Patologia do Instituto Nacional do Câncer (Dipat-Inca).

${ }_{3}^{2}$ Citogeneticista (MS) do Centro de Transplante de Medula Óssea do Instituto Nacional do Câncer (Cemo/Inca) - Rio de Janeiro-RJ.

${ }^{3}$ Médica. Professora Adjunta do Serviço de Hematologia do Hospital Universitário Pedro Ernesto - FCM-Uerj - Rio de Janeiro-RJ.

${ }_{5}^{4}$ Médica. Chefe do Serviço de Hematologia do Instituto Nacional do Câncer - Inca - Rio de Janeiro-RJ.

${ }^{5}$ Médico (MS) do Centro de Transplante de Medula Óssea do Instituto Nacional do Câncer (Cemo/Inca) - Rio de Janeiro-RJ.

${ }^{6}$ Citogeneticista. Pesquisadora Adjunta do Cemo/Inca e Professora do Curso de Pós-Graduação da Faculdade de Ciências Médicas

${ }^{7}$ Médica. Professora Adjunta do Departamento de Patologia e Laboratórios - FCM-Uerj e Pesquisadora do (Cemo-Inca) - Rio de Janeiro-RJ.

Universidade do Estado do Rio de Janeiro/ Instituto Nacional do Câncer - Rio de Janeiro-RJ. 


\section{Introdução}

A leucemia mieloide crônica (LMC) é uma doença mieloproliferativa clonal. Possui uma incidência de $15 \%$ a $20 \%$ de todas as leucemias no adulto e apresenta maior predominância no sexo masculino. A LMC foi a primeira neoplasia humana relacionada a uma alteração cromossômica. Em 1960, Nowell e Hungerford, estudando células de medula óssea de pacientes com LMC, observaram a presença de um pequeno cromossomo denominado Philadelphia (Ph) em 100\% dessas células, o que foi considerado um marco para a citogenética tumoral. Este rearranjo cromossômico corresponde a uma translocação recíproca envolvendo a região q34 do cromossomo 9, onde é mapeado o gene abl e a região q11 do cromossomo 22, onde é mapeado o gene bcr. A justaposição desses dois segmentos resulta na formação de um gene quimérico bcr-abl, cujo resultado é a formação de uma proteína de fusão bcr-abl (210 KDa) com atividade tirosino quinase aumentada, passando a transduzir sinais constitutivamente para a proliferação celular. ${ }^{1,2}$

O cromossomo Ph está presente em cerca de $95 \%$ dos pacientes com LMC. Esta doença progride através de três fases distintas caracterizadas por piora no quadro clínico e nas características laboratoriais. São elas: a fase crônica, a fase acelerada e a crise blástica. $\mathrm{O}$ avanço da doença torna $\mathrm{o}$ tratamento mais difícil a cada fase. Em $85 \%$ dos pacientes, o diagnóstico é realizado na fase crônica. ${ }^{1}$

Citogeneticamente, a fase crônica da LMC é caracterizada por uma única alteração cromossômica, o cromossomo $\mathrm{Ph}$. Quando a doença evolui, novas alterações cromossômicas são adicionadas ao clone $\mathrm{Ph}$. As anomalias cromossômicas mais comuns são: a aquisição de um novo cromossomo $\mathrm{Ph}$, trissomia de 8, isocromossomo do braço longo do 17 e trissomia do 19. A aquisição dessas alterações cromossômicas contribui para um aumento no potencial proliferativo e diminuição do potencial para expressão dos programas de diferenciação celular, caracterizando, portanto, a fase terminal da doença ou crise blástica. Dessa forma, a análise citogenética é importante para o diagnóstico, como fator prognóstico e monitorização da resposta terapêutica. ${ }^{2,3}$

Dentre as opções terapêuticas disponíveis para os pacientes com LMC estão a hidroxiureia, o interferon- $\alpha$, o transplante de células-tronco hematopoéticas (TCTH) e, mais recentemente, o imatinibe. A hidroxiureia é utilizada como terapêutica de escolha para pacientes refratários ou intolerantes ao interferon- $\alpha$. Embora a resposta hematológica tenha sido observada em mais de $90 \%$ dos pacientes, a resposta citogenética é raramente evidenciada, sendo considerada terapia paliativa. O interferon- $\alpha$ é mais indicado para a fase crônica da doença; no entanto, apenas 8\%-38\% dos pacientes com LMC apresentam resposta citogenética. ${ }^{4}$ Este tratamento apresenta uma toxicidade muito alta. Cerca de $80 \%$ dos pacientes apresentam sintomas gripais, fadiga, insônia, depressão, disfunção renal e pancitopenia, sendo que muitos pacientes precisam interromper o tratamento devido à intolerância. ${ }^{5}$ Apesar do TCTH ser, atualmente, o único tratamento que possibilita a cura desses pacientes, ele apresenta algumas complicações que levam ao aumento da mortalidade devido à doença enxerto contra hospedeiro, à imunossupressão e à toxicidade de múltiplos órgãos. Além disso, como a idade média do doente inicialmente acometido da LMC é de 40 a 50 anos, este fator, combinado com a ausência de doador histocompatível, limita a indicação de transplante a uma minoria de pacientes. ${ }^{6}$

Dessa forma, o tratamento com imatinibe representa uma nova expectativa de tratamento desses pacientes, por se tratar de um tratamento alvo molecular específico, impedindo a tirosino quinase BCR-ABL. Esta droga inibe seletivamente a proliferação e induz à apoptose nas linhagens celulares bcr-abl positivas. ${ }^{7} \mathrm{O}$ uso dessa medicação tem demonstrado resposta citogenética completa em 50\%-60\% dos pacientes em fase crônica após falência com interferon- $\alpha$ associada com aumento da sobrevida. ${ }^{8}$ Alguns trabalhos mostram a presença de efeitos adversos mínimos e bem tolerados pelos pacientes durante o tratamento, sendo os mais frequentes: cefaleia, náuseas/vômitos, diarreia e edema $.^{4-7} \mathrm{En}-$ tretanto, já foram descritas manifestações clínicas mais graves, podendo acarretar riscos de vida. ${ }^{9,10}$

O objetivo deste trabalho foi analisar a resposta citogenética e as manifestações clínicas adversas durante o tratamento com imatinibe em pacientes com LMC.

\section{Casuística e Método}

Cinquenta e um pacientes com LMC foram estudados clinicamente e através de revisão de prontuários entre janeiro de 2001 e dezembro de 2004. Estes pacientes foram incluídos no protocolo de imatinibe após terapia com hidroxiureia e resistência ao interferon- $\alpha$. Os pacientes foram diagnosticados nas Unidades de Hematologia do Hospital Universitário Pedro Ernesto e do Instituto Nacional de Câncer. O diagnóstico da LMC foi baseado na análise morfológica, em estudo molecular e citogenético, evidenciando a presença do cromossomo Ph. Este estudo foi realizado no momento do diagnóstico e durante o tratamento com imatinibe. Do total de pacientes estudados, 31 eram do sexo masculino (61\%) e 20 do sexo feminino (39\%), e a idade mediana era de 47 anos, com uma variação de 17 a 75 anos. O imatinibe foi administrado oralmente com a dose de $400 \mathrm{mg} /$ dia e, em quatro pacientes, esta dose foi aumentada para 600 a $800 \mathrm{mg} / \mathrm{dia}$ em virtude da ausência da resposta hematológica e citogenética nos três primeiros meses de acompanhamento. Os parâmetros de eficácia do medicamento foram avaliados a cada consulta para os aspectos clínicos e hematológicos. Este estudo foi aprovado pelo Comitê de Ética em Pesquisa, segundo as normas da resolução $\mathrm{n}^{\circ} 251$ sobre pesquisa envolvendo seres humanos, de 1997, do Conselho Nacional de Saúde no Hupe (906-CEP/Hupe), e no Inca pela 
resolução n ${ }^{\circ}$ 196, de 1996, do Conselho Nacional de Saúde (protocolo 58/05).

\section{Análise citogenética}

A análise citogenética foi realizada em células de medula óssea cultivadas em $80 \%$ de RPMI e $20 \%$ de soro fetal bovino a $37^{\circ} \mathrm{C}$. Duas horas antes do término da cultura foram adicionados $0,06 \mu \mathrm{g} / \mathrm{ml}$ de colchicina. Ao final da incubação, foi realizado o choque hipotônico $(\mathrm{KCl} 0,075 \mathrm{M})$ e o material foi fixado em metanol e ácido acético (3:1). A análise cromossômica foi realizada pela técnica de bandeamento GTG segundo Seabright (1971). ${ }^{11}$ O padrão cariotípico seguiu os critérios adotados pelo Sistema Internacional de Nomenclatura de Citogenética Humana (2001). ${ }^{12}$ Os pacientes foram analisados antes do tratamento com mesilato de imatinibe e duas a cinco vezes durante a terapia, com um acompanhamento de 6 a 42 meses. O número de células investigadas para cada paciente variou de 20 a 50 metáfases. A resposta citogenética foi definida de acordo com a porcentagem de células $\mathrm{Ph}$ positivas: resposta citogenética completa (RCC: $0 \%$ de células $\mathrm{Ph}$ ); resposta parcial (RCP: $1 \%$ a $34 \%$ de células $\mathrm{Ph}$ ), resposta citogenética maior (RCM: $\mathrm{RCC}+\mathrm{RCP}$ ); resposta citogenética menor (RCm: 35\%-95\% de células $\mathrm{Ph}$ ) e sem resposta citogenética (SRC: mais que $95 \%$ de células $\mathrm{Ph})^{5}$

\section{Análise estatística}

Os dados obtidos no presente estudo foram analisados utilizando-se estatística descritiva e analítica. Na estatística descritiva, os resultados foram apresentados utilizandose médias aritméticas, desvios-padrão, valores mínimos e máximos, frequências e porcentagens. Na estatística analítica foi empregada a análise univariada, visando verificar a associação entre as variáveis, através do teste não paramétrico do $\chi^{2}$ (qui-quadrado). Foi adotado o nível de significância de $5 \%$ de probabilidade $(\mathrm{p}=0,05)$ do resultado ser fruto do acaso. ${ }^{13}$

\section{Resultados}

Estudo clínico e citogenético em pacientes com LMC

Dos 51 pacientes, 29 (57\%) foram diagnosticados na fase crônica da doença, 17 na fase acelerada (33\%) e cinco em crise blástica (10\%). A análise citogenética mostrou, em 34 pacientes (66\%), a presença do cromossomo $\mathrm{Ph}$ como única alteração no início da terapia com imatinibe e 17 pacientes apresentaram alterações cromossômicas adicionais (34\%) (Tabela 1). A alteração cromossômica adicional mais frequente foi o ganho de um cromossomo $\mathrm{Ph}$, observado em cinco pacientes. Os estudos hematológicos mostraram que os pacientes que apresentaram somente o $\mathrm{Ph}$, a contagem média de células foi de $11,8 \times 10^{9} / 1$ para os leucócitos e de $319 \times 10^{9} / 1$ para as plaquetas, enquanto aqueles com alterações cromossômicas adicionais apresentavam maior
Tabela 1. Distribuição dos pacientes com LMC segundo a fase da doença e característica citogenética

\begin{tabular}{cccc}
\hline $\begin{array}{c}\text { Fases da } \\
\text { doença }\end{array}$ & $\begin{array}{c}\mathrm{N}^{\circ} \text { de } \\
\text { pacientes (\%) }\end{array}$ & \multicolumn{2}{c}{ Características citogenéticas } \\
\hline Crônica & $29(57 \%)$ & $25(49 \%)$ & $4(8 \%)$ \\
Acelerada & $17(33 \%)$ & $9(17 \%)$ & $8(16 \%)$ \\
Crise blástica & $5(10 \%)$ & $0(0 \%)$ & $5(10 \%)$ \\
Total & $51(100 \%)$ & $34(66 \%)$ & $17(34 \%)$ \\
\hline
\end{tabular}

número de leucócitos $\left(17,6 \times 10^{9} / 1\right)$ e menor contagem de plaquetas $\left(225 \times 10^{9} / 1\right)$.

\section{Manifestações clínicas e resposta citogenética}

A maioria dos pacientes (76\%) estudados apresentou manifestações clínicas adversas. Estas foram consideradas, na grande maioria, de grau leve a moderado. Cefaleia (35\%), náuseas (33\%), vômitos (31\%) e edema principalmente em membros inferiores (31\%) foram as mais comuns (Tabela 2).

Em relação à resposta citogenética, a RCM foi observada em vinte pacientes (39\%) (Tabela 3 ). Doze pacientes apresentaram resposta citogenética completa e se encontraram assintomáticos durante o período de coleta dos dados clínicos. Onze desses pacientes tinham apenas o cromossomo Ph e um permaneceu com uma alteração cromossômica adicional, a trissomia do 8 . O tempo médio para obtenção de resposta citogenética completa foi de 9,5 meses. A RCM foi observada em seis pacientes (12\%) e ausência de resposta citogenética foi observada em 25 (49\%). Neste último grupo, vale a pena ressaltar que cinco pacientes apresentaram duplo $\mathrm{Ph}$. Durante o tratamento, evolução citogenética clonal foi observada em 10,4\% dos pacientes. Durante o acompanhamento clínico, pacientes com alterações cromossômicas adicionais apresentaram uma sobrevida significantemente menor que os pacientes que apresentaram somente o $\mathrm{Ph}$ (14 meses versus 20 meses; $p=0,006$ ), independente do tipo de alteração cromossômica. Os pacientes que alcançaram RCM tiveram uma sobrevida maior que os pacientes SRC ( 27 meses versus 13 meses; $\mathrm{p}=0,007)$. Oito pacientes faleceram, sendo que seis com evolução da doença. Estes pacientes já tinham alterações cromossômicas adicionais antes do tratamento com imatinibe e não apresentaram resposta citogenética. Em relação aos outros dois pacientes, um faleceu por hemorragia pulmonar decorrente de aplasia medular e o outro por insuficiência respiratória de causa indeterminada.

\section{Discussão}

Estudos clínicos demonstraram a eficácia de mesilato de imatinibe no tratamento de pacientes com LMC, principalmente na fase crônica da doença. ${ }^{14,15}$ Os controles hematológico e citogenético apresentam resposta evidentemente 
Tabela 2. Principais manifestações clínicas observadas em pacientes com LMC tratados com mesilato de imatinibe

\begin{tabular}{|c|c|c|c|c|}
\hline \multirow[t]{2}{*}{ Distúrbios } & \multirow[t]{2}{*}{ Manifestações } & \multicolumn{3}{|c|}{ Número de pacientes (\%) } \\
\hline & & FA & FC & CB \\
\hline & Neutropenia & $1(3,44 \%)$ & $1(5,88 \%)$ & $0(0 \%)$ \\
\hline \multirow[t]{2}{*}{ Hematológicos } & Anemia & $9(30,96 \%)$ & $4(23,52 \%)$ & $2(40 \%)$ \\
\hline & Trombocitopenia & $3(10,32 \%)$ & $4(23,52 \%)$ & $0(0 \%)$ \\
\hline Psiquiátricos & Depressão & $1(3,44 \%)$ & $0(0 \%)$ & $0(0 \%)$ \\
\hline \multirow[t]{2}{*}{ Neurológicos } & Cefaleia & $8(27,52 \%)$ & $7(41,16 \%)$ & $4(80 \%)$ \\
\hline & Insônia & $3(10,32 \%)$ & $0(0 \%)$ & $0(0 \%)$ \\
\hline \multirow[t]{4}{*}{ Oculares } & Conjuntivite & $2(6,88 \%)$ & $2(11,76 \%)$ & $0(0 \%)$ \\
\hline & Fotofobia & $0(0 \%)$ & $0(0 \%)$ & $1(20 \%)$ \\
\hline & Hiperlacrimação & $0(0 \%)$ & $1(5,88 \%)$ & $1(20 \%)$ \\
\hline & Edema periorbital & $1(3,44 \%)$ & $2(11,76 \%)$ & $0(0 \%)$ \\
\hline Auditivos e Labirínticos & Vertigem & $4(13,76 \%)$ & $1(5,88 \%)$ & $2(40 \%)$ \\
\hline \multirow[t]{2}{*}{ Cardíacos } & Insuficiência cardíaca & $0(0 \%)$ & $1(5,88 \%)$ & $1(20 \%)$ \\
\hline & Taquicardia & $1(3,44 \%)$ & $1(5,88 \%)$ & $0(0 \%)$ \\
\hline \multirow[t]{6}{*}{ Trato grastrointestinal } & Náuseas & $11(37,84 \%)$ & $5(29,4 \%)$ & $3(60 \%)$ \\
\hline & Vômitos & $8(27,52 \%)$ & $5(29,4 \%)$ & $4(80 \%)$ \\
\hline & Diarreia & $3(10,32 \%)$ & $5(29,4 \%)$ & $1(20 \%)$ \\
\hline & Dor abdominal & $8(27,52 \%)$ & $4(23,52 \%)$ & $3(60 \%)$ \\
\hline & Odinofagia & $3(10,32 \%)$ & $1(5,88 \%)$ & $1(20 \%)$ \\
\hline & Plenitude gástrica & $1(3,44 \%)$ & $3(17,64 \%)$ & $0(0 \%)$ \\
\hline \multirow[t]{7}{*}{ Pele e mucosas } & Dermatite & $4(13,76 \%)$ & $7(41,16 \%)$ & $2(40 \%)$ \\
\hline & Mancha hipercrômica & $1(3,44 \%)$ & $1(5,88 \%)$ & $1(20 \%)$ \\
\hline & Prurido & $4(13,76 \%)$ & $2(11,76 \%)$ & $1(20 \%)$ \\
\hline & Petéquia & $0(0 \%)$ & $2(11,76 \%)$ & $1(20 \%)$ \\
\hline & Mucosite & $1(3,44 \%)$ & $2(11,76 \%)$ & $1(20 \%)$ \\
\hline & Edema facial & $3(10,32 \%)$ & $3(17,64 \%)$ & $1(20 \%)$ \\
\hline & Edema palpebral & $7(24,08 \%)$ & $2(11,76 \%)$ & $1(20 \%)$ \\
\hline \multirow[t]{4}{*}{ Músculo-esqueléticos } & Dor articular & $1(3,44 \%)$ & $0(0 \%)$ & $0(0 \%)$ \\
\hline & Dor óssea & $7(24,08 \%)$ & $4(23,52 \%)$ & $0(0 \%)$ \\
\hline & Cãimbra muscular & $5(17,2 \%)$ & $4(23,52 \%)$ & $0(0 \%)$ \\
\hline & Dor muscular & $1(3,44 \%)$ & $5(29,4 \%)$ & $0(0 \%)$ \\
\hline \multirow[t]{6}{*}{ Estado geral } & Febre & $8(27,52 \%)$ & $6(35,28 \%)$ & $3(60 \%)$ \\
\hline & Edema periférico & $7(24,08 \%)$ & $7(41,16 \%)$ & $3(60 \%)$ \\
\hline & Fadiga & $4(13,76 \%)$ & $4(23,52 \%)$ & $2(40 \%)$ \\
\hline & Fraqueza & $3(10,32 \%)$ & $5(29,4 \%)$ & $2(40 \%)$ \\
\hline & Calafrio & $1(3,44 \%)$ & $0(0 \%)$ & $1(20 \%)$ \\
\hline & Mal-estar & $0(0 \%)$ & $4(23,52 \%)$ & $1(20 \%)$ \\
\hline
\end{tabular}

FC: fase crônica; FA: fase acelerada; CB: crise blástica 
Tabela 3. Resposta citogenética durante o tratamento com mesilato de imatinibe em pacientes com LMC

\begin{tabular}{cc}
\hline Resposta citogenética & Pacientes (\%) \\
\hline RCM & $20(39 \%)$ \\
RCm & $6(12 \%)$ \\
SRC & $25(49 \%)$ \\
\hline Total & $51(100 \%)$ \\
\hline
\end{tabular}

RCM: resposta citogenética maior, de $1 \%$ a $34 \%$ de células $\mathrm{Ph}$ $\mathrm{RCm}$ : resposta citogenética menor, $35 \%-95 \%$ de células $\mathrm{Ph}$ SRC: sem resposta citogenética, mais que $95 \%$ de células $\mathrm{Ph}$

satisfatória em relação aos outros tratamentos disponíveis, incluindo resposta citogenética completa. ${ }^{14}$ Entretanto, estudos recentes têm mostrado que pacientes em uso dessa medicação geralmente apresentam algum tipo de efeito adverso. ${ }^{11,15}$ Como descrito por Kantarjian et al, ${ }^{14}$ o nosso estudo mostrou que os pacientes com resposta citogenética completa não apresentaram manifestações clínicas adversas e tiveram uma maior sobrevida em relação àqueles com resposta parcial e sem nenhuma resposta citogenética. Estes dois outros grupos de pacientes apresentaram efeitos adversos considerados leves/moderados. Os mais comuns foram cefaleia, náuseas, vômitos e edema em membros inferiores.

Em relação aos efeitos adversos, tem-se notado associação entre a dose do mesilato de imatinibe e sua tolerância (toxicidade) pelo organismo. As doses mais altas $(800 \mathrm{mg} /$ dia) têm mostrado associação com alterações clínicas adversas mais graves do que as doses mais baixas, como de $400 \mathrm{mg} /$ dia e $600 \mathrm{mg} / \mathrm{dia} .{ }^{11} \mathrm{O}$ aumento da dose do medicamento vem sendo associado a uma maior frequência de retenção de fluidos e erupções da pele. A dose limite de toxicidade, $1.000 \mathrm{mg} / \mathrm{dia}$, teve como efeitos mais evidentes edema, erupções de pele e citopenias. ${ }^{12}$

Entretanto, tem-se notado que o aumento da dose do medicamento se deve ao avanço natural do curso da doença para a fase acelerada, o que requer uma intervenção mais intensa. Nesse sentido, tem sido questionado se as alterações observadas seriam de fato causadas unicamente pelo uso do mesilato de imatinibe ou se a progressão da doença interferiria nos tipos de manifestações clínicas observadas nas altas dosagens. ${ }^{12,16}$ Mesmo com essa intervenção, não se obteve remissão da doença nos pacientes avaliados. Outros estudos evidenciaram remissão citogenética em pacientes com alta dose diária de mesilato de imatinibe $(800 \mathrm{mg} / \mathrm{dia})$, mas não avaliaram as manifestações adversas durante esse período. ${ }^{8}$ Portanto, são necessários novos estudos para avaliar a correlação da resposta citogenética e dos efeitos adversos em pacientes em uso de alta dose. A maioria dos pacientes $(57 \%)$ avaliados pelo presente estudo se encontrava na fase crônica da doença e recebia dosagens de $400 \mathrm{mg} /$ dia, o que está associado com reações adversas bem toleradas, compensando pelo benefício de ter a doença controlada progredindo lentamente para a fase acelerada.
O aparecimento de manifestações clínicas adversas devido ao uso do mesilato de imatinibe tem sido relacionado ao fato deste medicamento inibir algumas vias de sinalização, além da via BCL-ABL, como o receptor PDGF e c-kit. Dessa forma, a formação de edema, por exemplo, tem sido associada com a inibição da via do receptor PDGF, que regula a pressão do fluido intersticial por interações entre células do tecido conjuntivo e moléculas da matriz extracelular. ${ }^{9}$ Pacientes apresentando edema periférico apresentam boa resposta ao uso de diurético. Portanto, é recomendado que diuréticos devam ser iniciados quando for necessário prevenir edema pulmonar ou outras complicações. Raramente foram escritos casos de edema cerebral, articular, pleural e pericárdico. A maioria dos pacientes foi sensível ao tratamento com interrupção da medicação, diurético e otimização da função cardíaca com betabloqueador. ${ }^{17}$

Um dos distúrbios mais graves que podem surgir no tratamento com mesitato de imatinibe é a mielodepressão. Em alguns casos, os pacientes apresentam neutropenia, trombocitopenia e raramente anemia, segundo a literatura. ${ }^{18}$ Porém, na nossa amostra, esta última foi a alteração hematológica mais frequente, o que sugere a necessidade de investigação para excluir outras causas como a anemia ferropriva. Além disso, a mielodepressão compromete a resposta hematológica. Dependendo de sua gravidade, deve-se considerar a diminuição da dose ou mesmo a sua interrupção. ${ }^{18}$ No presente estudo houve um caso de aplasia medular de instalação súbita que levou ao óbito.

Em nosso estudo, as reações cutâneas ao mesilato de imatinibe foram relativamente frequentes. As mais observadas foram dermatite (25\%) e prurido (14\%). A fisiopatologia destas reações cutâneas ainda não está esclarecida. Ao contrário dos outros trabalhos, ${ }^{19,20}$ que mostraram que estes efeitos são dose dependentes, alguns dos pacientes (21\%) estudados que desenvolveram esta alteração estavam em uso de baixa dose, outros (4\%) usavam altas doses. Diante disto, estas reações podem estar mais relacionadas à hipersensiblidade do que ao efeito farmacológico direto. No entanto, uma casuística maior seria importante para este estudo, assim como estudos direcionados a farmacogenômica. Na evidência de um rash cutâneo, anti-histamínicos com ou sem esteroides tópicos e/ou sistêmicos são recomendados. Em casos mais graves, a terapia deve ser descontinuada e reintroduzida gradualmente com uso de esteroide. ${ }^{17}$

Alguns pacientes com LMC apresentaram uma resistência ao mesilato de imatinibe em células BCR-ABL positivas. Assim, deve haver um ou mais mecanismos intracelulares que levem à resistência ao medicamento. Nesses casos, na medula óssea, mesmo mediante o tratamento, células leucêmicas residuais com cromossomo $\mathrm{Ph}$ positivo podem ser encontradas. A provável causa desse efeito é que as células quiescentes sejam insensíveis à ação do medicamento. ${ }^{12,21}$ Entre as hipóteses levantadas, destacam-se a não inibição pelo medicamento do resíduo da tirosino quinase com ativi- 
dade $^{22}$ e a concentração intracelular reduzida de medicamento. ${ }^{23}$ Isso indica que o tema ainda tem que ser esclarecido por novos estudos, mas, como sugerem os resultados apresentados, a resistência ao imatinibe deve estar relacionada às alterações citogenéticas adicionais e a possíveis variações moleculares do cromossomo $\mathrm{Ph}$.

Durante o acompanhamento dos pacientes com LMC tratados com imatinibe a resposta citogenética tem um papel primordial. A análise citogenética em LMC apresenta três propósitos: (1) diagnóstico diferencial entre LMC e outras doenças mieloproliferativas; (2) monitorização da resposta terapêutica; (3) detecção de alterações cromossômicas adicionais ao $\mathrm{Ph}$ no diagnóstico ou durante o tratamento, acrescentando importantes informações em relação ao prognóstico do paciente..$^{23}$ Analisando nossos resultados, verificamos que, durante o acompanhamento clínico, pacientes com alterações cromossômicas adicionais apresentaram uma sobrevida significativamente menor que os pacientes que apresentaram somente o $\mathrm{Ph}(\mathrm{p}=0,006)$, independente do tipo de alteração cromossômica. Alguns estudos têm mostrado que alterações cromossômicas adicionais estariam relacionadas à resistência ao tratamento..$^{23}$

Dessa forma, o uso de mesilato de imatinibe representa uma nova expectativa de tratamento desses pacientes. Entretanto, é necessário caracterizar citogeneticamente e clinicamente os pacientes com LMC para poder predizer quais pacientes poderão se beneficiar com um tipo específico de tratamento, considerando a sua qualidade de vida e o tempo de sobrevida após a terapia. Isso é reforçado pelo fato de que alguns estudos têm demonstrado efeitos clínicos adversos que podem requerer tratamento farmacológico e suspensão do imatinibe. ${ }^{9,24}$

Mutações em domínios específicos da proteína BCRABL impedem a eficácia do imatinibe, o que caracteriza a resistência ao tratamento. Estes casos estimulam o desenvolvido de novas drogas alvo-moleculares, como o dasatinib, uma potente adenosina trifosfato (ATP) - inibidora do Src e de Abl quinases. Tal substância mostrou ação antiproliferativa em células de linhagem humana de LMC. ${ }^{25}$ Além do dasatinib, o nilotinib é uma outra nova droga derivada do imatinibe. É uma aminopiridina $20-50$ vezes mais potente que o imatinibe. ${ }^{26}$ Todas estas novas drogas têm o objetivo de serem mais potentes ou mais efetivas contra os clones BCR-ABL resistentes ao mesilato de imatinibe. No entanto, é importante o estudo das manifestações clínicas adversas observadas durante o tratamento com essas novas drogas, sendo necessário um estudo a longo prazo. ${ }^{27}$

Em relação ao tratamento de escolha para os pacientes com LMC, atualmente existem o transplante de células-tronco hematopoéticas (TCTH) e a terapia alvo-molecular. O TCTH é atualmente o único tratamento que proporciona a cura para os pacientes com LMC, porém, ele apresenta algumas complicações que levam ao aumento da mortalidade devido à doença-enxerto-contra-hospedeiro, à imunossupressão e à toxicidade de múltiplos órgãos. Além disso, a idade avançada do doente leva à comorbidade, e a ausência de doador histocompatível, limitando ainda mais a indicação do TCTH a uma minoria de pacientes. Por outro lado, o mesilato de imatinibe, uma droga alvo-molecular, tem mostrado resultados positivos para um grande número de pacientes, mas seu elevado custo faz com que apenas uma minoria dos pacientes tenha acesso a esse tratamento. Além disso, é importante considerar o aparecimento de manifestações clínicas de grau leve a grave, podendo acarretar a morte do paciente, assim como casos de resistência ao tratamento, sendo adotada uma nova forma de tratamento, uma terapia-alvo molecular mais potente, o dasatinibe, por exemplo. Nestes casos, a presença de alterações cromossômicas adicionais necessitam ser investigadas como um forte fator prognóstico, comparando a sobrevida dos pacientes que apresentam o cromossomo Philadelphia como única alteração cromossômica e os pacientes que apresentam alterações cromossômicas adicionais.

\section{Conclusões}

Os efeitos adversos são frequentes nos pacientes com leucemia mieloide crônica em uso de imatinibe em baixa dose, mas bem tolerados. Há necessidade de novos estudos para avaliação dos seus efeitos em altas doses a longo prazo. Dependendo do efeito adverso, deve-se realizar tratamento específico e evitar a suspensão da medicação, considerando o aumento da sobrevida e da remissão citogenética proporcionada pela medicação, exceto quando os riscos superam os benefícios.

Nossos resultados mostraram a importância do acompanhamento citogenético, onde a presença de alterações cromossômicas adicionais mostraram um comportamento biológico distinto; desta forma, a análise citogenética representa uma importante ferramenta para o diagnóstico e monitoramento deste grupo de pacientes.

\footnotetext{
Abstract

Chronic myeloid leukemia (CML) is a clonal myeloproliferative disorder characterized cytogenetically by the Philadelphia chromosome (Ph). Therapeutic options of this disease are: hydroxyurea, interferon- $\alpha$, allogeneic HSCT and more recently imatinib. This latter therapy demonstrated efficacy in the treatment of CML, particularly in the chronic phase. However some studies have demonstrated that there are additional chromosomal alterations related to resistance while others have reported undesirable clinical manifestations during imatinib therapy such as headache, nausea and vomiting. Because of the importance of this new molecular target therapy, it may be necessary to analyze the response of this treatment in respect to the quality of life of patients. The aim of this study was to analyze the clinical manifestations and the cytogenetic response during imatinib therapy in fifty-one patients with $C M L$ who had previously been treated using interferon- $\alpha$. Cytogenetic analysis was performed in bone marrow cells using GTG-banding.
} 
The commonest clinical manifestations were mild to moderate: headache (37\%), nausea (37\%), vomiting (33\%) and edema (33\%). Patients that achieved major cytogenetic response had a significantly longer median survival than patients without response $(p=0.007)$. Eight patients evolved to death; none of them exhibited cytogenetic responses to imatinib. Our results show the importance of the clinical (analyzing the degree of tolerance to the drug) and cytogenetic followup, where the presence of additional chromosomal alterations showed a distinct biological pattern that is not identifiable by molecular techniques, and so cytogenetic analysis is an important tool for the diagnosis and monitoring of this group of patientss. Rev. Bras. Hematol. Hemoter. 2010;32(2):116-122.

Key words: Leukemia, myeloid; signs and symptoms; cytogenetic analysis.

\section{Referências Bibliográficas}

1. Faderl S, Talpaz M, Estrov Z, OBrien S, Kurzrock R, Kantarji HM. The biology of chronic myeloid leukemia. N Engl J Med. 1999; 341(3):164-72.

2. Heim S, Mitelman F. Chronic Myeloid Leukemia. In: Heim S; Mitelman F. Cancer Cytogenetics. 2. ed. New York: Wiley-Liss, 1995. Cap 4; p. 33-68.

3. Schoch C, Haferlach T, Kern W, Schnittger S, Berger U, Hehlmann $\mathrm{R}$, et al. Occurrence of additional chromosome aberrations in chronic myeloid leukemia patients treated with imatinib mesylate. Leukemia. 2003;17(2):461-3.

4. Kantarjian HM, O'Brien S, Anderlini P, Talpaz M. Treatment of myelogenous leukemia-current status and investigational options. Blood. 1996;87(8):3069-81.

5. Goldman JM, Melo JV. Chronic myeloid leukemia - advances in biology and new approaches to treatment. N Engl J Med. 2003; 349(15):1451-64.

6. Deininger MW, Goldman JM, Lydon N, Melo JV. The tyrosine kinase inhibitor CGP 57148B selectively inhibits the growth of BCR-ABL positive cells. Blood. 1997;90(9):3691-8.

7. Druker BJ, Talpaz M, Resta DJ, Peng B, Buchdunger E, Ford JM, et al. Efficacy and safety of a specific inhibitor of BCR-ABL tyrosine kinase in chronic myeloid leukemia. N Engl J Med. 2001; 344(14):1031-7.

8. Jabbour E, Cortes J, OBrien S, Rios MB, Giles F, Kantarjian H. Management of patients with newly diagnosed chronic myeloid leukemia: opportunities and challenges. Clin Lymphoma Myeloma. 2007;7(S2):S51-7.

9. Ebnöether M, Stentoft J, Ford J, Buhl L, Gratwohl A. Cerebral oedema as a possible complication of treatment with imatinib. Lancet. 2002;359(9319):1751-2.

10. OBrien SG, Guilhot F, Larson RA, Gathmann I, Baccarani M, Cervantes $\mathrm{F}$, et al. Imatinib compared with interferon and lowdose cytarabine. N Engl J Med. 2003;348(11):994-1004.

11. Seabright M. A rapid banding technique for human chromosomes. Lancet. 1971;2(7731):971-2.

12. Deininger MW, OBrien SG, Ford JM, Druker BJ. Pratical Management of patients with chronic myeloid leukemia receiving imatinib. J Clin Oncol. 2003;21(8):1637-47.

13. Rodrigues PC. Bioestatística. $3^{\mathrm{a}}$ ed. Niterói: Eduff; 2002.

14. Kantarjian, H, Sawyers C, Hochhaus A, Guilhot F, Schiffer C, Gambacorti-Passerini C, et al. Hematologic and cytogenetic responses to imatinib mesylate in chronic myelogenous leukemia. N Engl J Med. 2002;346(9):645-52.
15. Funke VAM, Medeiro CR, Lima DH, Setúbal DC, Bitencourt MA, Bonfim CM, et al. Therapy of chronic myeloid leukemia with imatinib mesylate in Brazil: A study of 98 cases. Rev Bras Hematol Hemoter. 2005;27(3):159-65.

16. Ross DM, Hughes TP. Cancer treatment with kinase inhibitors: what have we learnt from imatinib? British Journal of Cancer. 2004;90(1):12-9.

17. Atallah E, Kantarjian H, Cortes J. Emerging safety with imatinib and other abl tyrosine kinase inhibitors. Clin Lymphoma Myeloma. 2007;7(S3):S105-12.

18. Ault P, Kantarjian H, Welch MA, Giles F, Rios MB, Cortes J. Interleukin 11 may improve thrombocytopenia associated with imatinib mesylate therapy in chronic myelogenous leukemia. Leuk Res. 2003;28(6):613-8.

19. Brouard M, Saurat JH. Cutaneous reactions to STI571. N Engl J Med. 2001;345(8):618-709.

20. Scheinfeld N. Imatinib mesylate and dermatology part 2: a review of the cutaneous side effects of imatinib mesylate. J Drugs Dermatol. 2006;5(3):228-31.

21. Swords R, Quinn J, Fay M, ODonnell R, Goldman J, Murphy PT. CML clonal evolution with resistance to single agent imatinib therapy. Clin Lab Hematol. 2005;27(2):347-9.

22. Goldman JM. Chronic myeloid leukemia - still a few questions. Ex Hematol. 2004;32(1):2-10.

23. Tauchi T, Ohyashisi K. The second generation of BCR-ABL tyrosine kinase inhibitors. Int J Hematol. 2006;83(4):294-300.

24. AlKindi S, Dennison D, Pathare A. Imatinib in pregnancy. Eur J Haematol. 2005;74(6):535-7.

25. OHare T, Pollock R, Stoffregen EP, Keats JA, Abdullah OM, Moseson EM, et al. Inhibition of wild-type and mutant Bcr-Abl by AP23464, a potent ATP-based oncogenic protein kinase inhibitor: implications for CML. Blood. 2004;104(8):2532-9.

26. Swords R, Alvarado Y, Giles F. Novel abl kinase inhibitors in chronic myeloid leukemia in blastic phase and philadelphia chomosome positive acute lymphoblastic leukemia. Clin Lymphoma Myeloma. 2007;7(S3):S113-9.

27. Goldman J, Apperley J, Kanfer E, Olavarria E, Marin D. Imatinib or transplant for chronic myeloid leukaemia? Lancet. 2003; 362(9378):172-3

Suporte Financeiro: Ministério da Saúde/Inca

Avaliação: Editor e dois revisores externos

Conflito de interesse: sem conflito de interesse

Recebido: 17/06/2009

Aceito: 04/08/2009 